\title{
Cyanuric Chloride as a Mild and Active Beckmann Rearrangement Catalyst
}

\author{
Yoshiro Furuya, Kazuaki Ishihara* and Hisashi Yamamoto* \\ Graduate School of Engineering, Nagoya University, Furo-cho, Chikusa-ku, Nagoya 464-8603, Japan, \\ and Department of Chemistry, The University of Chicago, 5735 South Ellis Avenue, Chicago, Illinois 60637
}

General Methods. Infrared (IR) spectra were recorded on a JASCO FT/IR 460 plus spectrometer. $\quad{ }^{1} \mathrm{H}$ NMR spectra were measured on a Varian Gemini-2000 spectrometer $(300 \mathrm{MHz})$ at ambient temperature. Data were recorded as follows: chemical shift in ppm from internal tetramethylsilane on the $\delta$ scale, multiplicity $(\mathrm{s}=\operatorname{singlet} ; \mathrm{d}=$ doublet; $\mathrm{t}=$ triplet; $\mathrm{q}=$ quartet, $\mathrm{m}=$ multiplet), coupling constant $(\mathrm{Hz})$, integration, and assignment. ${ }^{13} \mathrm{C}$ NMR spectra were measured on Varian Gemini-2000 (75 MHz) and VXR 500 (125 MHz) spectrometers. Chemical shifts were recorded in ppm from the solvent resonance employed as the internal standard (deuterochloroform at $77.00 \mathrm{ppm}$ ). All reaction were carried out under nitrogen. For thin-layer chromatography (TLC) analysis throughout this work, Merck precoated TLC plates (silica gel 60 GF254 0.25 mm or silica gel $\mathrm{NH}_{2}$ F254S $0.25 \mathrm{~mm}$ ) were used. The products were purified by column chromatography on silica gel (E. Merck Art. 9385). Microanalyses were performed at the Graduate School of Agriculture, Nagoya University. High resolution mass spectral analysis (HRMS) was performed at Chemical Instrument Center, Nagoya University. In experiments that required dry solvents, acetonitrile, nitromethane, toluene, and acetone were purchased from TCI or Wako as the "anhydrous" and stored over 4A molecular sieves. 1,4-Dioxane was freshly distilled from sodium. Other simple chemicals were analytical-grade and obtained commercially.

Representative Procedure for Preparing Ketoximes. To a solution of ketone (30 mmol) and 2.3 equiv of hydroxyamin hydrochloride $(69 \mathrm{mmol})$ in $40 \mathrm{~mL}$ of $\mathrm{MeOH}, 2.5$ equiv of sodium acetate trihydrate in $20 \mathrm{~mL}$ of water was added at room temperature, and the mixture was refluxed for $1 \mathrm{~h}$. After completion of reaction, the mixture was neutralized with saturated aqueous sodium hydrogen carbonate, and diluted with ether. The organic layer was separated, washed successively with brine and dried over anhydrous magnesium sulfate. The solvent was removed in vacuo, and the obtained product was purified in good yield by distillation or sublimation under reduced 
pressure.

Acetophenone Oxime (entry 1, Table 3): Purchased from TCI, Japan.

o-Methoxyacetophenone Oxime (entry 2, Table 3): ${ }^{1}$ IR (film) 3221, 2925, 1599, 1579, 1493, 1460, 1435, 1270, 1246, 1025, 917, $745 \mathrm{~cm}^{-1} ;{ }^{1} \mathrm{H}$ NMR $\left(300 \mathrm{MHz}, \mathrm{CDCl}_{3}\right) \delta 2.23$ (s, 3H), $3.83(\mathrm{~s}, 3 \mathrm{H}), 6.90-6.97(\mathrm{~m}, 2 \mathrm{H}), 7.28-7.34(\mathrm{~m}, 2 \mathrm{H}), 8.20(\mathrm{br}, 1 \mathrm{H})$.

m-Methoxyacetophenone Oxime (entry 3, Table 3): ${ }^{1}$ IR (film) 3250, 3076, 3003, 2938, $2835,1600,1579,1488,1464,1454,1429,1302,1231,1040,1006,942,855,783,692 \mathrm{~cm}^{-1} ;{ }^{1} \mathrm{H}$ NMR (300 MHz, $\left.\mathrm{CDCl}_{3}\right) \delta 2.29(\mathrm{~s}, 3 \mathrm{H}), 3.84(\mathrm{~s}, 3 \mathrm{H}), 6.91-6.95(\mathrm{~m}, 1 \mathrm{H}), 7.18-7.22(\mathrm{~m}, 2 \mathrm{H})$, 7.25-7.33 (m, 1H), 9.07 (br, 1H).

p-Methoxyacetophenone Oxime (entry 4, Table 3): ${ }^{1}$ IR (KBr) 3244, 2926, 1607, 1577, 1512, 1311, 1249, 1182, 1024, 922, 837, 822, $599 \mathrm{~cm}^{-1} ;{ }^{1} \mathrm{H}$ NMR (300 MHz, $\left.\mathrm{CDCl}_{3}\right) \delta 2.27$ (s, 3H), $3.83(\mathrm{~s}, 3 \mathrm{H}), 6.91(\mathrm{~d}, J=8.7 \mathrm{~Hz}, 2 \mathrm{H}), 7.58(\mathrm{~d}, J=8.7 \mathrm{~Hz}, 2 \mathrm{H}), 8.90(\mathrm{br}, 1 \mathrm{H})$.

p-Fluoroacetophenone Oxime (entry 5, Table 3): Purchased from Acros Organics.

1-(Benzo $[d][1,3]$ dioxol-5-yl)ethanone Oxime (entry 6, Table 3): ${ }^{1} \quad \mathrm{IR}(\mathrm{KBr}) 2922,1735$, 1498, 1448, 1302, 1228, 1036, 944, $807 \mathrm{~cm}^{-1} ;{ }^{1} \mathrm{H}$ NMR (300 MHz, $\mathrm{CDCl}_{3}$ ) $\delta 2.24$ (s, 3H), 5.99 (s, $3 \mathrm{H}), 6.81(\mathrm{~d}, J=8.1 \mathrm{~Hz}, 1 \mathrm{H}), 7.10(\mathrm{dd}, J=1.5,8.1 \mathrm{~Hz}, 1 \mathrm{H}), 7.18(\mathrm{~d}, J=1.5 \mathrm{~Hz}, 1 \mathrm{H}), 7.46(\mathrm{~s}, 1 \mathrm{H})$.

1-(Naphthalen-2-yl)ethanone Oxime (entry 7, Table 3): ${ }^{1}$ IR (KBr) 3215, 3053, 2911, 1597, 1503, 1448, 1420, 1384, 1307, 1196, 1132, 1017, 925, 825, $755 \mathrm{~cm}^{-1} ;{ }^{1} \mathrm{H}$ NMR $\left(300 \mathrm{MHz}, \mathrm{CDCl}_{3}\right)$

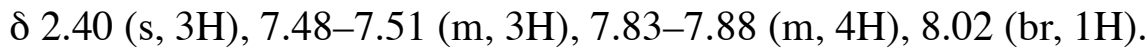

Isobutyrophenone Oxime (entry 8, Table 3): ${ }^{2}$ a cis- and trans-isomeric mixture (44:56); IR (KBr) 3267, 2968, 2930, 1456, 1384, 1314, 1019, 946, 770, $\left.700 \mathrm{~cm}^{-1} ; 1 \mathrm{H} \mathrm{NMR} \mathrm{(300} \mathrm{MHz,} \mathrm{CDCl}\right)_{3}$ $\delta 1.13(\mathrm{~d}, J=6.9 \mathrm{~Hz}, 3.36 \mathrm{H}), 1.21(\mathrm{~d}, J=7.2 \mathrm{~Hz}, 2.64 \mathrm{H}), 2.83$ (septet, $J=6.9 \mathrm{~Hz}, 0.56 \mathrm{H}), 3.60$ (septet, $J=7.2 \mathrm{~Hz}, 0.44 \mathrm{H}), 7.24-7.45$ (m, 5H), 9.30 (br, $0.56 \mathrm{H}), 9.65$ (br, $0.44 \mathrm{H})$.

Methyl 4-(Hydroxyimino)-4-phenylbutanoate: (entry 9, Table 3): ${ }^{3} \quad E / Z$ ratio $=90$ : 10; IR (film) 3404, 3057, 2952, 1737, 1497, 1439, 1284, 1201, 1174, 937, 762, $696 \mathrm{~cm}^{-1} ;{ }^{1} \mathrm{H}$ NMR (300 $\left.\mathrm{MHz}, \mathrm{CDCl}_{3}\right)$ for $(E)$-isomer $\delta$ 2.56-2.65 (m, 2H), 3.10-3.16 (m, 2H), $3.66(\mathrm{~s}, 3 \mathrm{H}), 7.57-7.40(\mathrm{~m}$, 3H), 7.59-7.63 (m, 2H), $8.81(\mathrm{br}, 1 \mathrm{H})$; ${ }^{1} \mathrm{H}$ NMR $\left(300 \mathrm{MHz}, \mathrm{CDCl}_{3}\right)$ for $(Z)$-isomer $\delta$ 2.57-2.66 (m, 2H), $2.89(\mathrm{t}, J=7.4 \mathrm{~Hz}, 2 \mathrm{H}), 3.68(\mathrm{~s}, 3 \mathrm{H}), 7.37-7.45(\mathrm{~m}, 3 \mathrm{H}), 7.60-7.63(\mathrm{~m}, 2 \mathrm{H})$

2-Decanone Oxime (entry 10, Table 3): ${ }^{4} \quad E / Z$ ratio $=76: 24$; IR $(\mathrm{KBr}) 3236,2925,2856$, 
1665, 1465, 1368, 1113, 956, $722 \mathrm{~cm}^{-1} ;{ }^{1} \mathrm{H}$ NMR (300 MHz, $\left.\mathrm{CDCl}_{3}\right)$ for $(E)$-isomer $\delta 0.86-0.90(\mathrm{~m}$, 3H), 1.27 (br, 10H), 1.47-1.52 (br, 2H), 1.86 (s, 3H), 2.17 (t, $J=7.5 \mathrm{~Hz}, 2 \mathrm{H}), 7.60-7.90$ (br, 1H); ${ }^{1} \mathrm{H}$ NMR (300 MHz, $\mathrm{CDCl}_{3}$ ) for $(Z)$-isomer $\delta$ 0.86-0.90 (m, 3H), 1.27 (br, 10H), 1.87 (s, 3H), 2.36 (t, $J=7.5 \mathrm{~Hz}, 2 \mathrm{H}), 7.60-7.90(\mathrm{br}, 1 \mathrm{H})$.

Diisopropylketone Oxime (entry 11, Table 3): $\quad$ IR (KBr) 3312, 2924, 1656, 1462, 1385, 1365, 1117, 1022, 934, 867, $762 \mathrm{~cm}^{-1} ;{ }^{1} \mathrm{H}$ NMR (300 MHz, $\left.\mathrm{CDCl}_{3}\right) \delta 1.13(\mathrm{~d}, J=6.9 \mathrm{~Hz}, 6 \mathrm{H}), 1.16$ $(\mathrm{d}, J=6.9 \mathrm{~Hz}, 6 \mathrm{H}), 2.57$ (septet, $J=6.9 \mathrm{~Hz}, 1 \mathrm{H}), 3.21$ (septet, $J=6.9 \mathrm{~Hz}, 1 \mathrm{H}), 9.72(\mathrm{br}, 1 \mathrm{H})$.

Cyclododecaonone Oxime (entry 12, Table 3): ${ }^{6}$ Purchased from Sigma-Aldrich Japan.

Cycloundecaonone Oxime (entry 13, Table 3): ${ }^{7} \quad \operatorname{IR}(\mathrm{KBr}) 2924,2859,1637,1474,935,729$ $\mathrm{cm}^{-1} ;{ }^{1} \mathrm{H}$ NMR $\left(300 \mathrm{MHz}, \mathrm{CDCl}_{3}\right) \delta 1.40-1.48(\mathrm{~m}, 12 \mathrm{H}), 1.62-1.70(\mathrm{~m}, 2 \mathrm{H}), 1.79$ (q, $J=6.3 \mathrm{~Hz}$, $2 \mathrm{H}), 2.27-2.36(\mathrm{~m}, 4 \mathrm{H})$.

Cyclodecaonone Oxime (entry 14, Table 3): ${ }^{7,8}$ IR (KBr) 3266, 2920, 2861, 1472, 1434, 949, $935 \mathrm{~cm}^{-1} ; 1 \mathrm{H} \mathrm{NMR}\left(\mathrm{CDCl}_{3}, 300 \mathrm{MHz}\right) \delta 1.42-1.59(\mathrm{~m}, 10 \mathrm{H}), 1.71-1.83(\mathrm{~m}, 4 \mathrm{H}), 2.44(\mathrm{t}, J=6.6$ $\mathrm{Hz}, 2 \mathrm{H})$.

Cyclooctaonone Oxime (entry 15, Table 3): Purchased from Sigma-Aldrich Japan.

Cyclohexaonone Oxime (entry 16, Table 3): Purchased from TCI, Japan.

\section{Representative Procedure for the Beckmann Rearrangement of Ketoximes (Table 3).}

The mixture of ketoxime $(2 \mathrm{mmol})$ and $5 \mathrm{~mol} \%$ of cyanuric chloride $9 \mathbf{a}(18.41 \mathrm{mg}, 0.1 \mathrm{mmol})$ in 4 $\mathrm{mL}$ of dry MeCN was refluxed. After completion of reaction, it was quenched with saturated aqueous sodium hydrogen carbonate. The organic layer was extracted with ethyl acetate, dried over anhydrous magnesium sulfate, and concentrated in vacuo. The crude product was purified by column chromatography on silica gel to give the corresponding amide in high yield.

Acetanilide (entry 1, Table 3): ${ }^{1}$ IR (KBr) 3293, 3155, 1657, 1597, 1555, 1500, 1434, 1369, 1322, 1264, 755, $695 \mathrm{~cm}^{-1} ;{ }^{1} \mathrm{H}$ NMR (300 MHz, $\left.\mathrm{CDCl}_{3}\right) \delta 2.17(\mathrm{~s}, 3 \mathrm{H}), 7.10$ (t, J = $\left.7.2 \mathrm{~Hz}, 1 \mathrm{H}\right)$, 7.26-7.36 (m, 2H), 7.40 (br, 1H), 7.50 (m, 2H).

o-Methoxyacetanilide (entry 2, Table 3): ${ }^{1}$ IR (KBr) 3250, 2924, 1654, 1541, 1496, 1458, 1252, 1117, 1025, $750 \mathrm{~cm}^{-1}$; ${ }^{1} \mathrm{H}$ NMR $\left(300 \mathrm{MHz}, \mathrm{CDCl}_{3}\right) \delta 2.20(\mathrm{~s}, 3 \mathrm{H}), 3.88(\mathrm{~s}, 3 \mathrm{H}), 6.87(\mathrm{dd}, J=$ 1.8, 8.1 Hz, 1H), 6.92-7.07 (m, 2H), $7.76(\mathrm{br}, 1 \mathrm{H}), 8.36$ (dd, $J=1.8,7.8 \mathrm{~Hz}, 1 \mathrm{H})$. 
m-Methoxyacetanilide (entry 3, Table 3): ${ }^{1} \quad \operatorname{IR}(\mathrm{KBr}) 3257,2924,2853,1664,1607,1562$, 1494, 1417, 1282, 1155, 1051, 860, 766, $688 \mathrm{~cm}^{-1} ;{ }^{1} \mathrm{H}$ NMR (300 MHz, $\mathrm{CDCl}_{3}$ ) $\delta 2.17$ (s, 3H), 3.80 (s, 3H), $6.61(\mathrm{dd}, J=2.1,8.0 \mathrm{~Hz}, 1 \mathrm{H}), 6.96(\mathrm{~d}, J=8.1 \mathrm{~Hz}, 1 \mathrm{H}), 7.18-7.28(\mathrm{~m}, 2 \mathrm{H}), 7.76(\mathrm{br}, 1 \mathrm{H})$.

p-Methoxyacetanilide (entry 4, Table 3): ${ }^{1}$ IR (KBr) 3242, 2924, 2853, 1743, 1647, 1606, 1512, 1246, 1030, $837 \mathrm{~cm}^{-1}$; ${ }^{1} \mathrm{H}$ NMR $\left(300 \mathrm{MHz}, \mathrm{CDCl}_{3}\right) \delta 2.16(\mathrm{~s}, 3 \mathrm{H}), 3.79(\mathrm{~s}, 3 \mathrm{H}), 6.86(\mathrm{~d}, J=$ $9.0 \mathrm{~Hz}, 2 \mathrm{H}), 7.07$ (brs, 1H), $7.39(\mathrm{~d}, J=9.0 \mathrm{~Hz}, 2 \mathrm{H})$.

p-Fluoroacetanilide (entry 5, Table 3): ${ }^{1} \quad$ IR (KBr) 3290, 3071, 2923, 2853, 1662, 1617, 1559, 1507, 1402, 1235, $836 \mathrm{~cm}^{-1} ;{ }^{1} \mathrm{H}$ NMR (300 MHz, $\left.\mathrm{CDCl}_{3}\right) \delta 2.17(\mathrm{~s}, 3 \mathrm{H}), 7.01(\mathrm{~d}, J=9.0 \mathrm{~Hz}, 2 \mathrm{H})$, 7.18 (brs, 1H), 7.45 (dd, $J=4.8,9.0 \mathrm{~Hz}, 2 \mathrm{H})$.

3,4-Methyledioxyacetanilide (entry 6, Table 3): ${ }^{1}$ IR (KBr) 3326, 2923, 1742, 1662, 1546, 1486, 1444, 1238, 1181, 1039, $814 \mathrm{~cm}^{-1} ;{ }^{1} \mathrm{H}$ NMR (300 MHz, $\left.\mathrm{CDCl}_{3}\right) \delta 2.15$ (s, 3H), 5.95 (s, 2H), 6.74-6.75 (m, 2H), $7.11(\mathrm{~b}, 1 \mathrm{H}), 7.16(\mathrm{~d}, J=1.2 \mathrm{~Hz}, 1 \mathrm{H})$.

$N$-(2-Naphthyl)acetamide (entry 7, Table 3): $\quad$ IR (KBr) 3283, 1668, 1589, 1561, 1471, 1396, 1351, 1281, 858, 816, $746 \mathrm{~cm}^{-1}$; ${ }^{1} \mathrm{H}$ NMR (300 MHz, $\left.\mathrm{CDCl}_{3}\right) \delta 2.24$ (s, 3H), 7.40-7.46 (m, 3H), 7.76-7.80 (m, 4H), 8.18 (br, 1H).

$N$-Isopropylbenzamide (entry 8, Table 3): ${ }^{9} \quad \operatorname{IR}(\mathrm{KBr}) 3299,2971,1632,1347,1289,1170$, 1137, $696 \mathrm{~cm}^{-1} ;{ }^{1} \mathrm{H}$ NMR $\left(300 \mathrm{MHz}, \mathrm{CDCl}_{3}\right) \delta 1.26(\mathrm{~d}, J=6.6 \mathrm{~Hz}, 6 \mathrm{H}), 4.28(\mathrm{~m}, 1 \mathrm{H}), 6.11(\mathrm{br}, 1 \mathrm{H})$, 7.38-7.51 (m, 3H), 7.74-7.78 (m, 2H).

$N$-Phenylisobutyramide (entry 8, Table 3): ${ }^{2} \quad$ IR (KBr) 3433, 3302, 2968, 1661, 1600, 1549, 1442, 1309, 758, $695 \mathrm{~cm}^{-1} ;{ }^{1} \mathrm{H}$ NMR $\left(300 \mathrm{MHz} \mathrm{CDCl}_{3}\right) \delta 1.26(\mathrm{~d}, J=6.9 \mathrm{~Hz}, 6 \mathrm{H}), 2.51$ (septet, $\mathrm{J}=$ $6.9 \mathrm{~Hz}, 1 \mathrm{H}), 7.08-7.55(\mathrm{~m}, 5 \mathrm{H}), 7.15(\mathrm{br}, 1 \mathrm{H})$.

Methyl 4-Oxo-4-(phenylamino)butanoate (entry 9, Table 3): ${ }^{1}$ IR (KBr) 3360, 2924, 2853, $1724,1685,1597,1543,1500,1445,1404,1365,1312,1244,1216,1167,761,700 \mathrm{~cm}^{-1} ;{ }^{1} \mathrm{H}$ NMR $\left(300 \mathrm{MHz}, \mathrm{CDCl}_{3}\right) \delta 2.67(\mathrm{t}, J=6.6 \mathrm{~Hz}, 2 \mathrm{H}), 2.77(\mathrm{t}, J=6.6 \mathrm{~Hz}, 2 \mathrm{H}), 3.72(\mathrm{~s}, 3 \mathrm{H}), 7.10-7.52(\mathrm{~m}$, 5H), 7.55 (brs, $1 \mathrm{H})$.

N-Octylacetamide (entry 10, Table 3): ${ }^{1}$ IR (film) 3289, 3088, 2956, 2856, 1652, 1558, 1465, 1438, 1370, 723, $603 \mathrm{~cm}^{-1}$; ${ }^{1} \mathrm{H}$ NMR $\left(300 \mathrm{MHz} \mathrm{CDCl}_{3}\right) \delta 0.88(\mathrm{t}, J=6.3 \mathrm{~Hz}, 3 \mathrm{H}), 1.27$ (br, 10H), 1.44-1.51 (m, 2H), 1.97 (s, 3H), 3.19-3.26 (m, 2H), 5.49 (br, 1H).

$N$-Isopropylisobutyramide (entry 11, Table 3): ${ }^{10}$ IR (KBr) 3297, 2970, 2933, 2873, 1644, 
1551, 1366, 1244, 1179, 1096, $\left.699 \mathrm{~cm}^{-1} ;{ }^{1} \mathrm{H} \mathrm{NMR} \mathrm{(300} \mathrm{MHz,} \mathrm{CDCl}_{3}\right) \delta 1.14(\mathrm{~d}, J=6.9 \mathrm{~Hz}, 12 \mathrm{H})$, 2.28 (septet, $\mathrm{J}=6.9 \mathrm{~Hz}, 1 \mathrm{H}), 4.02-4.13(\mathrm{~m}, 1 \mathrm{H}), 5.23(\mathrm{br}, 1 \mathrm{H})$.

Azacyclotridecan-2-one (entry 12, Table 3): ${ }^{1}$ IR (film) 3299, 2931, 2854, 1639, 1546, 1448, $1059 \mathrm{~cm}^{-1} ;{ }^{1} \mathrm{H}$ NMR $\left(300 \mathrm{MHz}, \mathrm{CDCl}_{3}\right) \delta 1.32$ (br, 14H), 1.48-1.51 (m, 2H), 1.64-1.70 (m, 2H), $2.18-2.22(\mathrm{~m}, 2 \mathrm{H}), 3.31(\mathrm{dd}, J=6.0,10.8 \mathrm{~Hz}, 2 \mathrm{H})$.

Azacyclododecan-2-one (entry 13, Table 3): ${ }^{11}$ IR (KBr) 3313, 2926, 1639, 1545, $1466 \mathrm{~cm}^{-1}$; ${ }^{1} \mathrm{H}$ NMR $\left(300 \mathrm{MHz}, \mathrm{CDCl}_{3}\right) \delta$ 1.26-1.47 (m, 12H), 1.54-1.74 (m, 4H), 2.20-2.25 (m, 2H).

Azacycloundecan-2-one (entry 14, Table 3): ${ }^{12}$ IR (KBr) 3307, 2929, 1638, 1551, 1463, 689 $\mathrm{cm}^{-1} ;{ }^{1} \mathrm{H}$ NMR $\left(300 \mathrm{MHz}, \mathrm{CDCl}_{3}\right.$ ) $\delta$ 1.32-1.78 (m, 14H), 2.18-2.23 (m, 2H), 3.33 (dd, $J=5.4,11.1$ $\mathrm{Hz}, 2 \mathrm{H})$.

Azacyclononan-2-one (entry 15, Table 3): ${ }^{1} \quad \operatorname{IR}(\mathrm{KBr}) 3313,2927,1646,1541,1447,1155$, 1031, 797, $730 \mathrm{~cm}^{-1} ;{ }^{1} \mathrm{H}$ NMR (300 MHz, $\left.\mathrm{CDCl}_{3}\right) \delta$ 1.56-1.63 (m, 8H), 1.80-1.88 (m, 2H), $2.44(\mathrm{t}$, $J=6.3 \mathrm{~Hz}, 2 \mathrm{H}), 3.35-3.38(\mathrm{~m}, 2 \mathrm{H}), 5.63(\mathrm{br}, 1 \mathrm{H})$.

E-Caprolactam (entry 16, Table 3): ${ }^{1}$ IR (KBr) 3211, 3075, 2928, 2856, 1654, 1486, 1438, 1417, 1365, 1198, 1125, $823 \mathrm{~cm}^{-1} ;{ }^{1} \mathrm{H}$ NMR $\left(300 \mathrm{MHz}, \mathrm{CDCl}_{3}\right) \delta 1.62-1.80(\mathrm{~m}, 6 \mathrm{H}), 2.47(\mathrm{t}, J=$ $5.7 \mathrm{~Hz}, 2 \mathrm{H}), 3.21(\mathrm{t}, J=6.3 \mathrm{~Hz}, 2 \mathrm{H}), 6.05(\mathrm{br}, 1 \mathrm{H})$.

Preparation of $\boldsymbol{O}$-2,4,6-Tris(1-phenylethylideneaminooxy)-1,3,5-triazine (5a). To a stirred solution of acetophenone oxime $(2.04 \mathrm{~g}, 15 \mathrm{mmol})$ and $\mathbf{9 a}(922 \mathrm{mg}, 5 \mathrm{mmol})$ in $10 \mathrm{~mL}$ of dry $\mathrm{MeCN}$, triethylamine $(2.23 \mathrm{~mL}, 16 \mathrm{mmol})$ was added at room temperature. After 5a was precipitated, the white solid was separated by filtration, and washed successively with ether, water and dried under reduced pressure ( $>90 \%$ yield). Mp. $155-156{ }^{\circ} \mathrm{C}$; IR (KBr) 2923, 1578, 1552 , 1459, 1355, 1105, 1077, 886, 755, $691 \mathrm{~cm}^{-1} ;{ }^{1} \mathrm{H}$ NMR (300 MHz, $\mathrm{CDCl}_{3}$ ) $\delta 2.56$ (s, 9H), 7.43-7.47 (m, 9H), $7.85(\mathrm{~d}, J=8.1 \mathrm{~Hz}, 6 \mathrm{H}) ;{ }^{13} \mathrm{C} \mathrm{NMR}\left(125 \mathrm{MHz}, \mathrm{CDCl}_{3}\right) \delta 14.8$ (3C), 127.3 (6C), $128.6(6 \mathrm{C})$, 130.8 (3C), 134.8 (3C), 163.4 (3C), 174.5 (3C). Anal. Calcd for $\mathrm{C}_{27} \mathrm{H}_{24} \mathrm{~N}_{6} \mathrm{O}_{3}$ : C, 67.49; H, 5.03; N, 17.49; Found: C, 67.04; H, 4.91; N, 17.23.

\section{References}

(1) Commercially available.

(2) Luca, L. D.; Giacomelli, G.; Porcheddu, A. J. Org. Chem. 2002, 67, 6272.

(3) Imoto, H.; Sugiyama, Y.; Kimura, H.; Momose, Y. Chem. Pharm. Bull. 2003, 51, 138. 
(4) Yukawa, Y.; Saeki, M.; Suzuki, S. Bull. Chem. Soc. Jpn. 1966, 39, 2266.

(5) Hawkes, G. E.; Herwig, K.; Roberts, J. D. J. Org. Chem. 1974, 39, 1017.

(6) Groth, P. Acta Chem. Scand., Series A: Phy. and Inorg. Chem. 1979, A33, 503.

(7) Müller, E.; Padeken, H. G.; Salamon, M.; Fiedler, G. Chem. Ber. 1965, 98, 1893.

(8) Bank, S.; Closson, W. D. Tetrahedron 1968, 24, 381.

(9) Gajda, T.; Zwierzak, A. Synthesis 1981, 1005.

(10) Fache, F.; Jacquot, L.; Lemaire, M. Tetrahedron Lett. 1994, 35, 3313.

(11) Olah, G. A.; Fung, A. P. Synthesis 1979, 537.

(12) Williams, D. E.; Craig, K. S.; Patrick, B.; McHardy, L. M.; Soest, R. v.; Roberge, M.; Andersen, R. J. J. Org. Chem. 2002, 67, 245. 